\title{
On the derivation of second order variable step variable order block backward differentiation formulae for solving stiff ODEs
}

\begin{abstract}
In this paper, we derive a second order Variable Step Variable Order Block Backward Differentiation Formulae (VSVO-BBDF (2)). This method pertains to the study of solving stiff Ordinary Differential Equations (ODEs) of second order ( $\left.y^{\prime \prime}\right)$. The pertinent findings in term of total number of steps, accuracy and computational time are displayed and discussed through distinct tables.
\end{abstract}

Keyword: BBDF methods; Second order ODEs; Stiff ODEs 